\title{
The Assignment of Scores Procedure for Ordinal Categorical Data
}

\author{
Han-Ching Chen and Nae-Sheng Wang \\ Department of Statistics, Feng Chia University, Taichung City 407, Taiwan \\ Correspondence should be addressed to Nae-Sheng Wang; nswang@fcu.edu.tw
}

Received 25 February 2014; Accepted 18 August 2014; Published 11 September 2014

Academic Editor: Chaowei Yang

Copyright ( 2014 H.-C. Chen and N.-S. Wang. This is an open access article distributed under the Creative Commons Attribution License, which permits unrestricted use, distribution, and reproduction in any medium, provided the original work is properly cited.

Ordinal data are the most frequently encountered type of data in the social sciences. Many statistical methods can be used to process such data. One common method is to assign scores to the data, convert them into interval data, and further perform statistical analysis. There are several authors who have recently developed assigning score methods to assign scores to ordered categorical data. This paper proposes an approach that defines an assigning score system for an ordinal categorical variable based on underlying continuous latent distribution with interpretation by using three case study examples. The results show that the proposed score system is well for skewed ordinal categorical data.

\section{Introduction}

Ordinal data often occur during sampling survey and experimental design; therefore, it is difficult to get the interval data. The obtained data are usually "categorical data" or "ordinal categorical data," which are collected based on a scale of "strongly agree," "agree," "have no opinion," "disagree," and "strongly disagree." Because most data in traditional statistical methods are interval data, researchers often assign these ordinal categorical data a score first, convert them into interval data, and then conduct further statistical analyses, such as factor analysis, principal analysis, and discriminate analysis.

One method of assigning a score to these ordinal categorical data is to assign a score to ordinal categorical data subjectively (e.g., 5 for strongly agree, 4 for agree, 3 for no opinion, 2 for disagree, and 1 for strongly disagree). However, the original scale is an ordinal scale, without the concept of distance. After assigning a score from 5 to 1 , the scale becomes an interval scale and thus has the concept of distance. The distance between strongly agree (5) and no opinion (3) is the same as that between agree (4) and disagree (2), which exaggerates the information provided by the data. Other score-assignment methods assign the data-generated scores objectively. These methods include the Ridit score relatively to an identified distribution [1], the Conditional Median under a given cumulative distribution function [2], Conditional Mean scoring functions based on the underlying distribution [3], and the normal scores [4]. In many applications, treating the latent variable models for ordinal categorical data requires the Bayesian model to calculate parameters [5]. Another two score-assignment methods can be referred to in testing for $2 \times k$ ordered tables. For processing this problem of the sensitivity of the linear rank test on the scores, Kimeldorf et al. suggested the min-max scoring [6] and Gautam et al. suggested the iso-chi-square approach for the $2 \times k$ ordered tables [7]. However, this approach may be detailed and involves complex computations of the prime assumption.

This paper aims to provide an alternative scoring system based on an underlying continuous latent variable to determine the scores of ordinal categorical data and explain the results by using three examples. The remainder of this paper is organized as follows: Section 2 introduces the scoring system and relevant theories; Section 3 describes how scores are assigned to ordinal categorical data, the main theorem, and the relevant corollary; Section 4 gives three examples to explain the effects of scoring results with the formula of Theorem 1; and lastly, Section 5 offers a conclusion 
and provides suggestions on score assignment for ordinal categorical data. Some property details are provided in the Appendix.

\section{The Scoring System}

For an ordinal categorical random variable $Y$ with the probabilities $\left(p_{1}, \ldots, p_{k}\right), k$ denotes the number of categories. A scoring system is a systematic method for assigning numerical values to ordinal categories [8].

The scores are computed from $\left(p_{1}, \ldots, p_{k}\right)$. Let $s_{j}=$ $h_{k}\left(j, p_{1}, \ldots, p_{k}\right)$ be the scores assigned to the $j$ th category, and let $S=\left\{h_{k}\left(j, p_{1}, \ldots, p_{k}\right)\right\}$ denote the scoring system determined by the scoring functions $h_{k}\left(j, p_{1}, \ldots, p_{k}\right)$.

For ordinal categorical data, Bross introduced a scoring system, which he called Ridit scores [1]. Let $\pi_{j}=\sum_{i \leq j} p_{i}$. Bross defended the Ridit score for category $j$ by $r_{j}=$ $(1 / 2)\left(\pi_{j-1}+\pi_{j}\right)$. Brockett defended a Conditional Median Score under $G[2]$, where $G$ denotes some given cumulative distribution functions selected either in accordance with some theoretical latent distribution of the categorical variable under study or in accordance with the desirable properties for the planned method of analysis. For example, if the categorical variable represents income levels, $G$ may represent a Pareto family distribution function. Let $s_{j}=h_{k}\left(j, p_{1}, \ldots, p_{k}\right)$ represent the scores assigned and let $F$ be the cumulative distribution function corresponding to this scoring system (i.e., $F\left(s_{j}\right)=\pi_{j}$ ). Brockett found a scoring system $\left\{s_{j}\right\}, s_{j}=$ $G^{-1}\left(r_{j}\right), j=1, \ldots, k$, that satisfies the distance and minimizes $d(F, G)=\max _{x}|F(x)-G(x)|$, where $r_{j}$ is the Ridit score for the category $j$ (Figure 1 ).

Fielding suggested a scoring function $f_{j}$ based on the conditional mean of a category, assuming that the data are generated by an assumed distributional form $G$ [3]. Consider the following:

$$
\begin{aligned}
f_{j} & =\frac{\left\{\int_{G^{-1}\left(\pi_{j-1}\right)}^{G^{-1}\left(\pi_{j}\right)} u g(u) d u\right\}}{p_{j}} \\
& =\frac{\left\{\int_{\pi_{j-1}}^{\pi_{j}} G^{-1}(u) d u\right\}}{p_{j}}, \quad j=1, \ldots, k .
\end{aligned}
$$

The next section will introduce a scoring system based on given cumulative distribution function satisfying some condition.

\section{Scoring Procedure for Ordinal Categorical Data}

For an ordinal categorical random variable $Y$ with the probabilities $\left(p_{1}, \ldots, p_{k}\right), k$ denotes the number of categories. Let an unobserved continuous variable underlie $Y$ [9], and let $Z$ denote the underlying latent variable. Suppose that $-\infty=$ $c_{0}<c_{1}<\cdots<c_{j}<\cdots<c_{k}=\infty$ are cut points of the continuous scale such that the observed response $Y$ satisfies

$$
Y=a_{j} \quad \text { if } c_{j-1}<Z \leq c_{j} .
$$

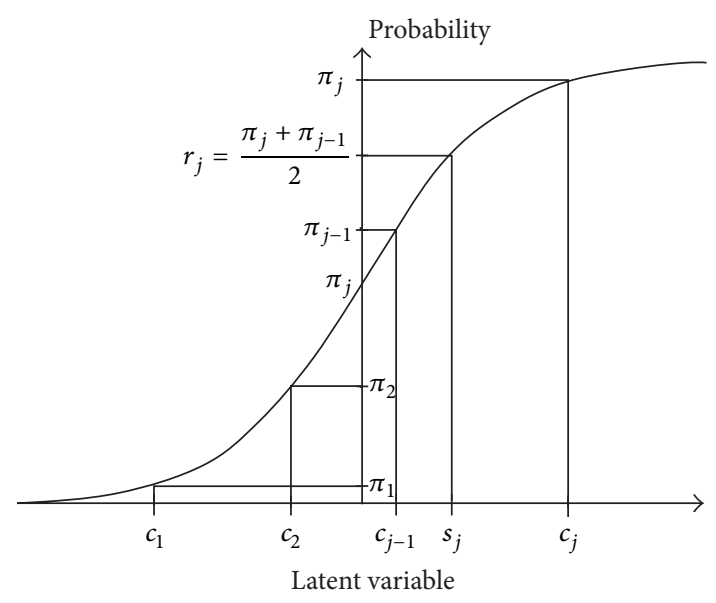

FIGURE 1: The correlation plot of $s_{j}$ and $r_{j}$.

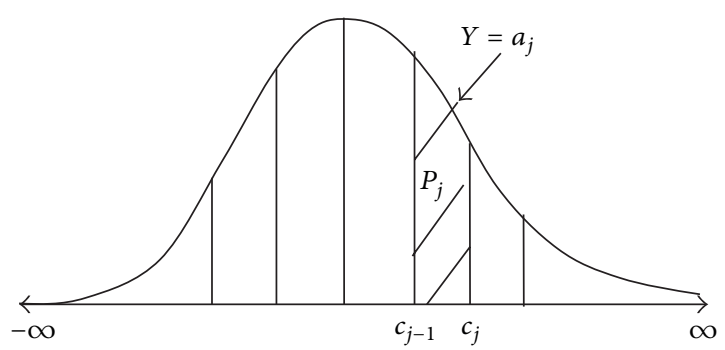

FIgURE 2: The plot of assigned score $a_{j}$ and underlying latent variable.

In other words, $Y$ falls in assigned score $a_{j}$ when the latent variable falls in the $j$ th interval of values (Figure 2). This section introduces a scoring system for $Y$ based on the underlying latent variable of $Z$ satisfying $E Y=E Z$.

Theorem 1. Let $Y$ be an ordinal categorical response variable with the probabilities $\left(p_{1}, \ldots, p_{k}\right)$, where $k$ denotes the number of categories. Assume that $Z$ is a continuous underlying distribution of $Y$ with the distribution function of $G$ and probability density function $g$ and assume that EZ exists. Suppose that $-\infty=c_{0}<c_{1}<\cdots<c_{j}<\cdots<c_{k}=\infty$ are cut points of $Z$. For each $j=1, \ldots, k$, let $a_{j}$ denote the score of $Y$ and $Y=a_{j}$ if and only if $c_{j-1}<Z \leq c_{j}$. If one takes $a_{j} \equiv E\left\{Z \mid c_{j-i}<Z \leq c_{j}\right\}$, then one has

$$
\begin{aligned}
& \text { (a) } E Y=E Z \text {; } \\
& \text { (b) } a_{j}=E\left(G^{-1}\left(\pi_{j-1}+\left(\pi_{j}-\pi_{j-1}\right) U\right)\right) \text {, } \\
& \text { where } U \sim U(0,1) \text {; } \\
& \text { (c) } c_{j}=G^{-1}\left(\pi_{j}\right) \text {. }
\end{aligned}
$$

Corollary 2. If the underlying distribution $Z$ is $U(0,1)$, then $a_{j}=r_{j}$, where $r_{j}$ is the Ridit score. 
Corollary 3. Let $r_{j}=(1 / 2)\left(\pi_{j-1}+\pi_{j}\right)$ (Ridit score), then one has $a_{j} \approx G^{-1}\left(r_{j}\right)$, where

$$
a_{j}=E\left(G^{-1}\left(\pi_{j-1}+\left(\pi_{j}-\pi_{j-1}\right) U\right)\right), \quad U \sim U(0,1) .
$$

Appendix shows the proofs of all the properties.

Remark 4. Assume that $Z$ is a continuous underlying distribution of $Y$ with the distribution function of $G$ is known; therefore, the cut point $c_{j}$ does not need to be given in advance.

Remark 5. The score $a_{j}$ defined in this study fulfills Brockett's Postulate 2 (Branching Property) [2]: suppose there are more than two categories, and for statistical or computation reasons we wish to combine two adjacent categories. In this case, the scores of the unaffected categories remain unchanged. Symbolically, if the $i$ and $(i+1)$ st categories are combined, then

$$
\begin{array}{r}
h_{k-1}\left(t, p_{1}, \ldots, p_{i-1}, p_{i}+p_{i+1}, p_{i+2}, \ldots, p_{k}\right) \\
\quad= \begin{cases}h_{k}\left(t, p_{1}, \ldots, p_{k}\right) & t \leq i-1 \\
h_{k}\left(t+1, p_{1}, \ldots, p_{k}\right) & t \geq i+1 .\end{cases}
\end{array}
$$

This postulate states that there is consistency within the scoring system as $k$ changes.

Remark 6. Agresti introduced a score $v_{j}$, and let $v_{j}=\Phi^{-1}\left(r_{j}\right)$, where $\Phi$ is a cumulative distribution function for standard normal distribution and $r_{j}$ is the Ridit score in category $j$ [4]. Then, by Corollary 3 , when $G=\Phi$, we have $a_{j} \approx v_{j}$.

\section{Examples}

Example 1. This example is a prospective study of maternal drinking and congenital malformations [10]. Table 1 presents a summary of the questionnaire results for alcohol consumption as completed by women who have passed their first trimester. Results show whether the newborns suffered from congenital malformations after birth. The average number of drinks per day was used to measure alcohol consumption, which was an explanatory variable of an ordinal categorical nature.

This study examines the correlation between the mothers' level of alcohol consumption and congenital malformation in newborns. The traditional approach is to use a contingency table. However, this study assigns scores to the level of alcohol consumption and uses a statistical value $M^{2}=(n-1) r^{2}$ to test the correlation, where $r$ is a coefficient of correlation. The square root of $M^{2}$ has an approximately standard normal distribution under the null hypothesis. The $P$ value is the right-tail probability above the observed value [11]. Different assigned scores are used to calculate the $M^{2}$ and the $P$ value. As Table 2 shows, the values of $M^{2}$ and $P$ value of the method by midpoints $P$ value of 0.0104 and the proposed method with exponential score have the significant $P$ value of 0.018572 , indicating that they are close to each other, whereas the
TABLE 1: Presence or absence of congenital sex organ malformation categorized by alcohol consumption of the mother [10].

\begin{tabular}{lccccc}
\hline \multirow{2}{*}{ Malformation } & \multicolumn{5}{c}{ Alcohol consumption (average \# drinks/day) } \\
& 0 & $<1$ & $1-2$ & $3-5$ & $\geqq 6$ \\
\hline Absent & 17066 & 14464 & 788 & 126 & 37 \\
Present & 48 & 38 & 5 & 1 & 1 \\
\hline Total & 17114 & 14502 & 793 & 127 & 38 \\
\hline
\end{tabular}

midpoints and midranks (Ridit score) have a large difference. And the proposed method with lognormal score $P$ value of 0.002318 has the smallest significant $P$ values that indicates it is well fit for this skewed data.

In this case, Graubard and Korn noted that the results of the trend test applied to this data set are sensitive to the choice of scores and the $P$ value for equally spaced scores is 0.1764 . The Ridit score gave a $P$ value of 0.5533 . Using the midpoints scores, we found the $P$ values corresponding to the exponential score value are close to each other [10]. Therefore, we suggest that using the proposed method with exponential scores or lognormal score could be well in this example.

Example 2. This example is from Agresti, who used several data sets from the General Social Survey (GSS) [4]. Table 3 shows the results of 2,387 responses from the GSS to a question on whether heaven exists where the data presents a skewed property.

Table 4 presents a comparison of the results to examine the proposed normal scores based on Ridits with the method of Remark 5 and the formula of Theorem 1 . As in Table 4, the Agresti normal score $v_{j}$ and the proposed normal score $a_{j}$ are close. This table also shows the proposed score $a_{j}$, including the exponential, logistic, and lognormal scores. The computation for scores is illustrated. Let $\pi_{j}$ be the cumulative relative frequency; that is, $\pi_{1}=0.648, \pi_{2}=0.856, \pi_{3}=0.942, \pi_{4}=1.0$ and $\pi_{0}=0$. Then, we apply function (b) in (3) of Theorem 1 to compute the score value $a_{j}$ with distribution $G$ to be standard normal, exponential, logistic, and lognormal, respectively. The result also indicates the relatively larger gap in lognormal score that has good fit for this skewed data.

Example 3. This example is from Snedecor and Cochran [12]. In this example, patients with leprosy were divided into those with little infiltration and those with much infiltration, based on a measure of a certain type of skin damage. Their health status was also classified into five levels after the 48week treatment (Table 5). This study uses the formula of Theorem 1 and that proposed by Fielding to assign scores and to compare the results [3]. As Table 6 shows, the values are close to each other. In addition, Figures 3(a)-3(d) show the results of scores under the different distribution with the formula of Theorem 1. The distribution pattern in these figures shows that the shapes of the scores computed from different underlying distribution are different. 
TABLE 2: Alternative scoring systems for ordinal categories with exact one-sided $P$ values.

\begin{tabular}{|c|c|c|c|c|c|}
\hline & \multicolumn{5}{|c|}{ Alcohol consumption (average \# drinks/day) } \\
\hline & 0 & $<1$ & $1-2$ & $3-5$ & $\geqq 6$ \\
\hline Midpoints & 0 & 0.5 & 1.5 & 4.0 & 7.0 \\
\hline \multirow[t]{2}{*}{ Standardized } & -0.9 & -0.72 & -0.38 & -0.48 & 1.52 \\
\hline & \multicolumn{5}{|c|}{$M^{2}=6.570134 \quad P$ value $=0.0104(*)$} \\
\hline Equally spaced & 1.0 & 2.0 & 3.0 & 4.0 & 5.0 \\
\hline \multirow{2}{*}{ Standardized } & -1.26 & -0.63 & 0.00 & 0.63 & 1.26 \\
\hline & \multicolumn{5}{|c|}{$M^{2}=1.827816 \quad P$ value $=0.1764$} \\
\hline Midranks & 8557.5 & 24365.5 & 32013.0 & 32473.0 & 32555.5 \\
\hline \multirow[t]{2}{*}{ Standardized } & -1.69 & -0.16 & 0.58 & 0.63 & 0.63 \\
\hline & \multicolumn{5}{|c|}{$M^{2}=0.351438 \quad P$ value $=0.2860$} \\
\hline Ridit score & 0.262694 & 0.747989 & 0.982762 & 0.996884 & 0.999417 \\
\hline \multirow[t]{2}{*}{ Standardized } & -1.68566 & -0.15734 & 0.582024 & 0.626497 & 0.634473 \\
\hline & \multicolumn{5}{|c|}{$M^{2}=0.351438 \quad P$ value $=0.5533$} \\
\hline Normal score & -0.63502 & 0.668423 & 2.116563 & 2.739277 & 3.253699 \\
\hline \multirow{2}{*}{ Standardized } & -0.64932 & 0.58412 & 1.660698 & 2.141198 & 2.550635 \\
\hline & \multicolumn{5}{|c|}{$M^{2}=1.455888 \quad P$ value $=0.113793$} \\
\hline Exponential score & 0.304753 & 1.378283 & 4.060658 & 5.771211 & 7.446831 \\
\hline \multirow[t]{2}{*}{ Standardized } & -1.17343 & -0.81223 & 0.090276 & 0.665807 & 1.229585 \\
\hline & \multicolumn{5}{|c|}{$M^{2}=4.343807 \quad P$ value $=0.018572(*)$} \\
\hline Logistic score & -1.03201 & 1.087917 & 4.04327 & 5.76809 & 7.446247 \\
\hline \multirow[t]{2}{*}{ Standardized } & -1.30621 & -0.69014 & 0.168719 & 0.669972 & 1.157663 \\
\hline & \multicolumn{5}{|c|}{$M^{2}=2.220069 \quad P$ value $=0.068113$} \\
\hline Lognormal score & 0.529903 & 1.950675 & 8.285174 & 15.41468 & 25.71126 \\
\hline \multirow[t]{2}{*}{ Standardized } & -0.94672 & -0.81014 & -0.20121 & 0.484139 & 1.473942 \\
\hline & \multicolumn{5}{|c|}{$M^{2}=8.01653 \quad P$ value $=0.002318(*)$} \\
\hline
\end{tabular}

${ }^{*}$ Significant at $5 \%$.

TABLE 3: Responses about belief in heaven [11].

\begin{tabular}{lccccc}
\hline & Definitely & Probably & $\begin{array}{c}\text { Probably } \\
\text { not }\end{array}$ & $\begin{array}{c}\text { Definitely } \\
\text { not }\end{array}$ & Total \\
\hline Count & 1546 & 498 & 205 & 138 & 2387 \\
Proportion & 0.648 & 0.208 & 0.086 & 0.058 & 1.0 \\
Ridit score & 0.324 & 0.752 & 0.899 & 0.971 & \\
\hline
\end{tabular}

\section{Conclusion}

In this paper, we provide alternative methods of assigning scores to ordinal categorical variables based on the underlying continuous distribution. These procedures are simpler and easier ways to assign scores. We cite three real case studies to explain the process and results of the calculations and propose that the score systems for ordinal variables are easy to perform, effective, and operationally useful, similar to the Ridit score or Agresti scores.

The Equal Space or Rank methods are generally used as scores (i.e., midranks or Ridit scores in Example 1) for processing ordinal categorical data. However, if the data are right-skewed or left-skewed or if some categories have many more observations than other categories, the result is obviously poor. This paper uses several underlying distributions as the alternatives of scores (e.g., the $M^{2}$ obtained from exponential score is closest to the midpoint in Example 1). We propose that if underlying distribution exists, these methods are also helpful for improving the development of traditional statistical techniques and software applications. By the three illustrations, this study suggests that the lognormal score can be applied well when the ordinal categorical data is skewed, and the normal score may be used when the data is relatively balanced among categories.

There are many methods for processing ordinal categorical data. However, not all of these methods require scoreassignment methods (e.g., Cumulative Logit Models and Proportional Odds Models) to convert ordinal categorical data into interval data for analysis.

However, if independent variables are categorical ordinal variables, they are considered categorical data and processed as dummy variables in the traditional (general) statistical method. In addition, if many response variables exist in categorical ordinal variables, it is advisable to assign scores to variables to convert them into an interval scale for further statistical analysis. The benefits of this process to independent variables are as follows: (1) the degree of freedom is 1 (which is $k-1$ originally) and (2) the characteristics of ordinals can also be used, indicating that related computational analysis for variables may be less complicated. 
TABLE 4: The results of responses about belief in heaven with different formulas.

\begin{tabular}{lcccc}
\hline & Definitely & Probably & Probably not & Definitely not \\
\hline Count & 1546 & 498 & 205 & 138 \\
Proportion & 0.648 & 0.209 & 0.086 & 0.058 \\
Agrestic normal score $v_{j}$ & -0.457 & 0.681 & 1.277 & 1.897 \\
Normal score $a_{j}$ & -0.45699 & 0.680765 & 1.277267 & 1.897112 \\
Exponential score $a_{j}$ & 0.391322 & 1.394286 & 2.295073 & 3.543686 \\
Logistic score $a_{j}$ & -0.73619 & 1.109254 & 2.188874 & 3.514354 \\
Lognormal score $a_{j}$ & 0.633184 & 1.975389 & 3.586822 & 6.666614 \\
\hline
\end{tabular}

TABLE 5: 196 patients classified according to change in health and degree of infiltration [12].

\begin{tabular}{lccccc}
\hline Degree of infiltration & & Change in health & & Total \\
& Marked & $\begin{array}{c}\text { Improvement } \\
\text { Moderate }\end{array}$ & Slight & Stationary & Worse \\
\hline Little & 11 & 27 & 42 & 53 & 11 \\
Much & 7 & 15 & 16 & 13 & 1 \\
\hline Total & 18 & 42 & 58 & 66 & 12 \\
\hline
\end{tabular}

TABLE 6: Results of using different formulae under the same distribution scores.

\begin{tabular}{|c|c|c|c|c|c|}
\hline & Worse & Stationary & Slight & Moderate & Marked \\
\hline Total frequencies & 12 & 66 & 58 & 42 & 18 \\
\hline Proportions & 0.061224 & 0.336735 & 0.295918 & 0.214286 & 0.091837 \\
\hline Ridit scores & $\begin{array}{l}0.030612 \\
(0.03043)\end{array}$ & $\begin{array}{c}0.229592 \\
(0.228588)\end{array}$ & $\begin{array}{c}0.545918 \\
(0.545036)\end{array}$ & $\begin{array}{c}0.80102 \\
(0.800381)\end{array}$ & $\begin{array}{c}0.954082 \\
(0.953808)\end{array}$ \\
\hline Normal scores & $\begin{array}{c}-1.87187 \\
(-1.83849)\end{array}$ & $\begin{array}{c}-0.74019 \\
(-0.73567)\end{array}$ & $\begin{array}{c}0.115356 \\
(0.114767)\end{array}$ & $\begin{array}{c}0.845272 \\
(0.839809)\end{array}$ & $\begin{array}{l}1.685788 \\
(1.66233)\end{array}$ \\
\hline Logistic scores & $\begin{array}{l}-3.45526 \\
(-3.78093)\end{array}$ & $\begin{array}{l}-1.21062 \\
(-1.3212)\end{array}$ & $\begin{array}{c}0.184192 \\
(0.18643)\end{array}$ & $\begin{array}{l}1.392684 \\
(1.439734)\end{array}$ & $\begin{array}{c}3.033884 \\
(3.338361)\end{array}$ \\
\hline Lognormal scores & $\begin{array}{c}0.153836 \\
(0.146667)\end{array}$ & $\begin{array}{c}0.477022 \\
(0.481287)\end{array}$ & $\begin{array}{l}1.122272 \\
(1.15068)\end{array}$ & $\begin{array}{c}2.32861 \\
(2.444555)\end{array}$ & $\begin{array}{c}5.396699 \\
(6.654603)\end{array}$ \\
\hline
\end{tabular}

\section{Appendix}

\section{Proof of Proposition in Section 3}

Proof of Theorem 1. Since $Y=a_{j}$ if and only if $c_{j-1}<Z \leq c_{j} \quad$ Since, for each $j=1, \ldots, k$, thus,

$$
\begin{gathered}
p_{j}=P\left(Y=a_{j}\right)=P\left(c_{j-1}<Z \leq c_{j}\right) \\
\pi_{j}=P\left(Y \leq a_{j}\right)=P\left(Z \leq c_{j}\right)=G\left(c_{j}\right) .
\end{gathered}
$$

We have $c_{j}=G^{-1}\left(\pi_{j}\right)$.

And

$$
\begin{aligned}
E Y & =\sum_{j} a_{j} P\left(Y=a_{j}\right) \\
& =\sum_{j} E\left(Z \mid c_{j-1}<Z<c_{j}\right) P\left(Y=a_{j}\right)
\end{aligned}
$$

$$
=\sum_{j} E\left(Z \mid Y=a_{j}\right) P\left(Y=a_{j}\right)=E(E(Z \mid Y))=E Z .
$$

$$
\begin{aligned}
a_{j} & =E\left(Z \mid c_{j-1}<Z \leq c_{j}\right) \\
& =\frac{1}{P\left(c_{j-1}<Z<c_{j}\right) \int_{c_{j-1}}^{c_{j}} z g(z) d z} \\
& =\frac{\int_{G\left(c_{j-1}\right)}^{G\left(c_{j}\right)} G^{-1}(w) d w}{G\left(c_{j}\right)-G\left(c_{j-1}\right)} \\
& =\frac{\int_{\pi_{j-1}}^{\pi_{j}} G^{-1}(w) d w}{\pi_{j}-\pi_{j-1}} \\
& =\int_{0}^{1} G^{-1}\left(\pi_{j-1}+\left(\pi_{j}-\pi_{j-1}\right) U\right) d u
\end{aligned}
$$




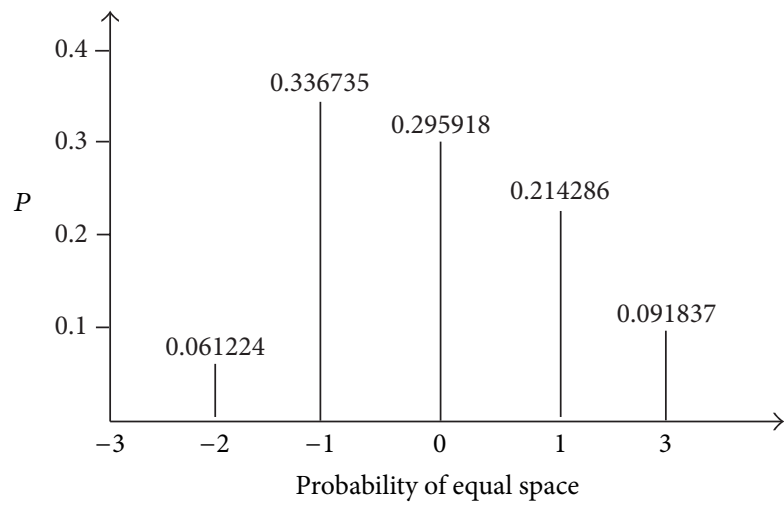

(a)

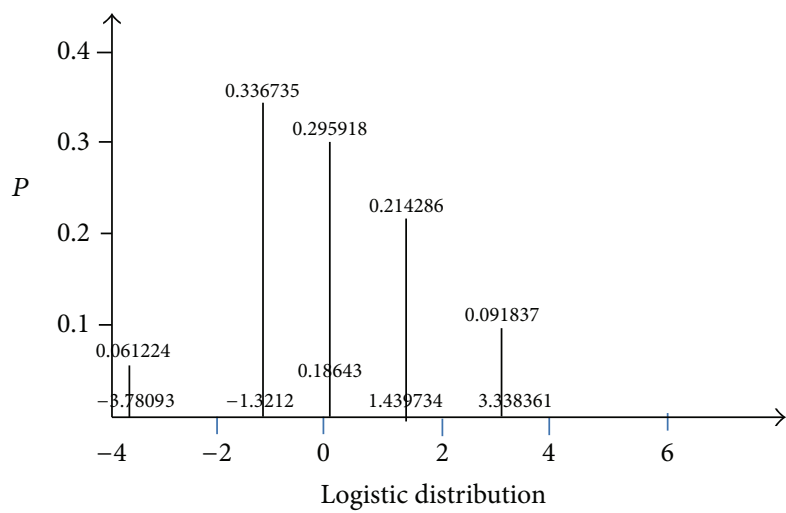

(c)

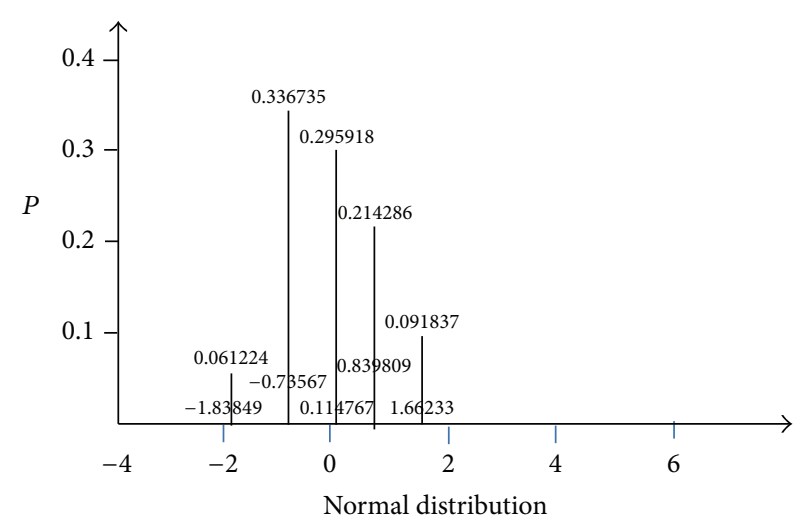

(b)

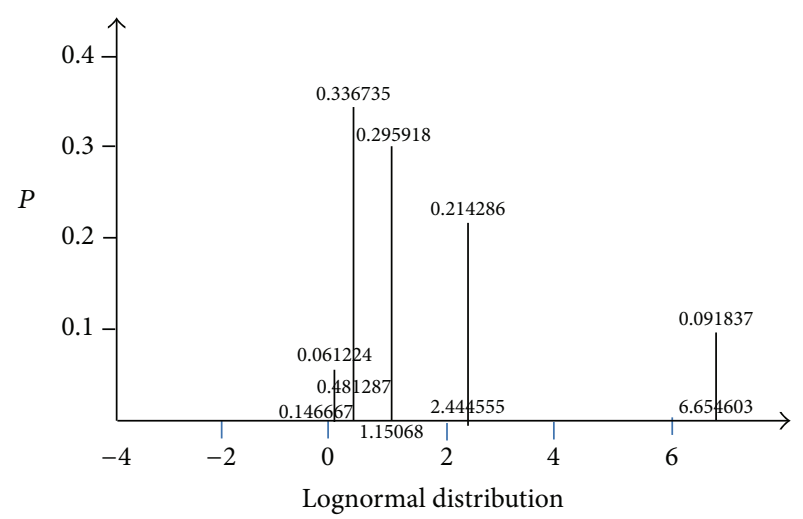

(d)

FIGURE 3: Proability plots comparing the results of scores under the different distributions with the formula of Theorem 1. (a) Equal Space, (b) normal distribution, (c) logistic distribution, and (d) lognormal distribution.

$$
=E\left(G^{-1}\left(\pi_{j-1}+\left(\pi_{j}-\pi_{j-1}\right) U\right)\right)
$$

thus, we have $a_{j}=E\left(G^{-1}\left(\pi_{j-1}+\left(\pi_{j}-\pi_{j-1}\right) U\right)\right)$, where $U \sim$ $U(0,1)$.

Proof of Corollary 2. Since $Z \sim U(0,1)$, thus we have $G(z)=$ $z$ and $G^{-1}(z)=z$.

By

$$
\begin{aligned}
a_{j} & =E\left(G^{-1}\left(\pi_{j-1}+\left(\pi_{j}-\pi_{j-1}\right) U\right)\right) \\
& =E\left(\pi_{j-1}+\left(\pi_{j}-\pi_{j-1}\right) U\right) \\
& =\frac{\pi_{j-1}+\left(\pi_{j}-\pi_{j-1}\right)}{2} \\
& =\frac{\left(\pi_{j}+\pi_{j-1}\right)}{2} \\
& =r_{j}
\end{aligned}
$$

thus, we have $a_{j}=r_{j}$ if $Z \sim U(0,1)$.
Proof of Corollary 3. Let $E(Z)=\mu$; we have $G^{-1}(z)=$ $G^{-1}(\mu)+\left(1 / g\left(G^{-1}(\mu)\right)\right)(z-\mu)+o(z-\mu)$; then $E G^{-1}(Z) \approx$ $G^{-1}(E Z)$.

By Theorem 1 , since $a_{j}=E\left(G^{-1}\left(\pi_{j-1}+\left(\pi_{j}-\pi_{j-1}\right) U\right)\right)$, where $U \sim U(0,1)$, thus, we have

$$
\begin{aligned}
a_{j} & \approx G^{-1}\left(\pi_{j-1}+\left(\pi_{j}-\pi_{j-1}\right) E U\right) \\
& =G^{-1}\left(\pi_{j-1}+\left(\pi_{j}-\pi_{j-1}\right) \frac{1}{2}\right) \\
& =G^{-1}\left(\frac{\pi_{j-1}+\pi_{j}}{2}\right)=G^{-1}\left(r_{j}\right) .
\end{aligned}
$$

\section{Conflict of Interests}

The authors declare that there is no conflict of interests regarding the publication of this paper. 


\section{References}

[1] I. D. Bross, "How to use ridit analysis," Biometrics, vol. 14, pp. 19-20, 1958.

[2] P. L. Brockett, "A note on the numerical assignment of scores to ranked categorical data," Journal of Mathematical Sociology, vol. 8, no. 1, pp. 91-101, 1981.

[3] A. Fielding, "Scoring functions for ordered classifications in statistical analysis," Quality and Quantity, vol. 27, no. 1, pp. 117, 1993.

[4] A. Agresti, Analysis of Ordinal Categorical Data, Wiley, New York, NY, USA, 2nd edition, 2010.

[5] P. McCullagh, "Regression models for ordinal data," Journal of the Royal Statistical Society B, vol. 42, no. 2, pp. 140-142, 1980.

[6] G. Kimeldorf, A. R. Sampson, and L. R. Whitaker, "Min and max scorings for two-sample ordinal data," Journal of the American Statistical Association, vol. 87, no. 417, pp. 241-247, 1992.

[7] S. Gautam, A. R. Sampson, and H. Singh, "Iso-chi-squared testing of $2 \times k$ ordered tables," Canadian Journal of Statistics, vol. 29, pp. 609-619, 2001.

[8] L. L. Golden and P. L. Brockett, "The effect of alternative scoring methods on the analysis of rank order categorical data," Journal of Mathematical Sociology, vol. 12, no. 4, pp. 383-389, 1987.

[9] J. A. Anderson and P. R. Philips, "Regression, discrimination and measurement models for ordered categorical variables," Journal of the Royal Statistical Society. Series C: Applied Statistics, vol. 30, no. 1, pp. 22-31, 1981.

[10] B. I. Graubard and E. L. Korn, "Choice of column scores for testing independence in ordered $2 \times k$ contingency tables," Biometrics, vol. 43, no. 2, pp. 471-476, 1987.

[11] A. Agresti, An Introduction to Categorical Data Analysis, Wiley Series in Probability and Statistics, John Wiley \& Sons, New York, NY, USA, 2nd edition, 2007.

[12] G. W. Snedecor and W. G. Cochran, Statistical Methods, 6th edition, 1975. 

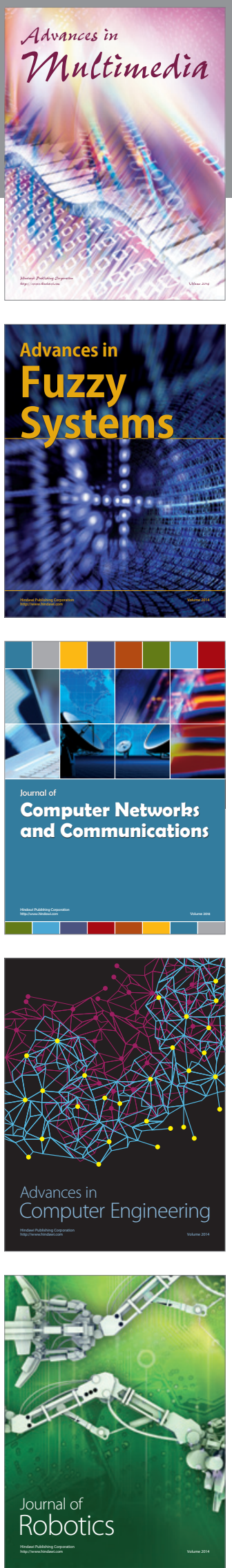

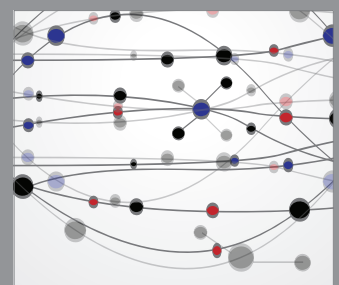

The Scientific World Journal
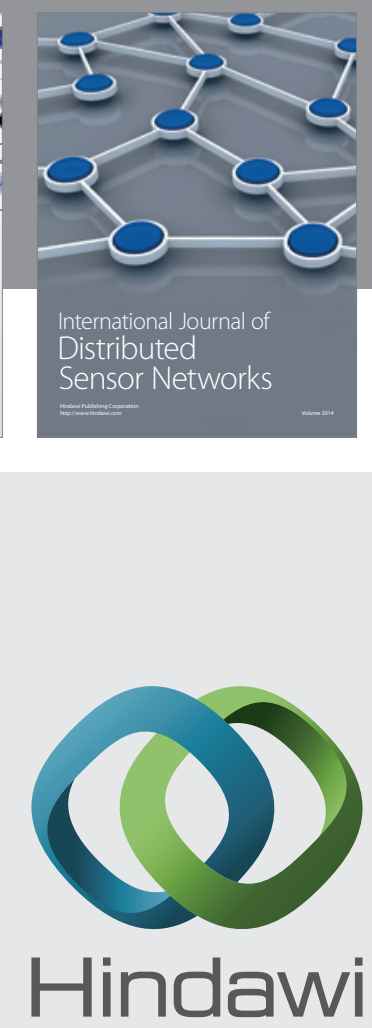

Submit your manuscripts at

http://www.hindawi.com
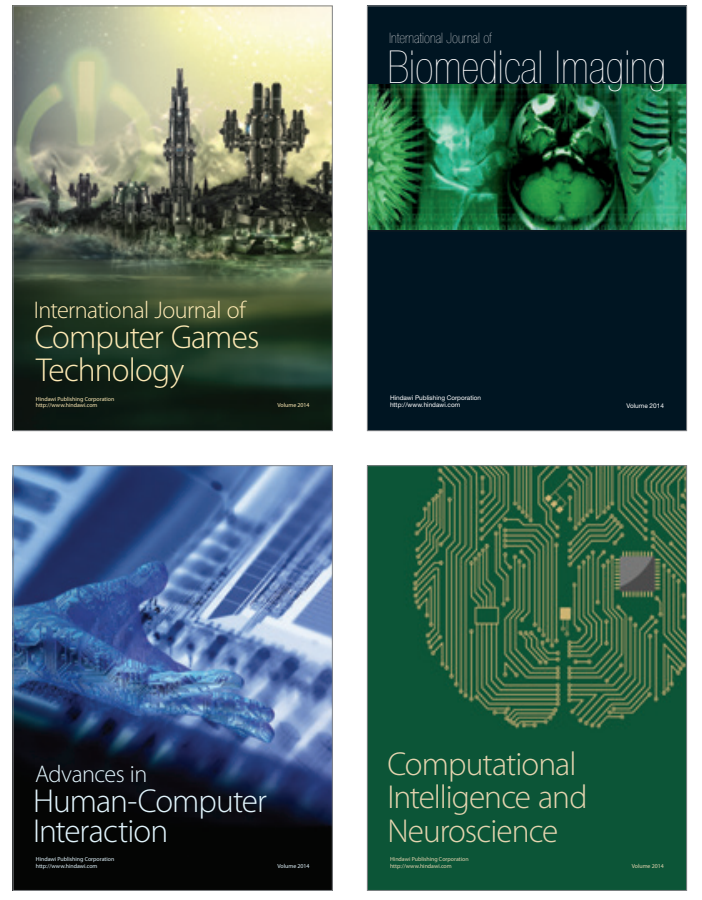
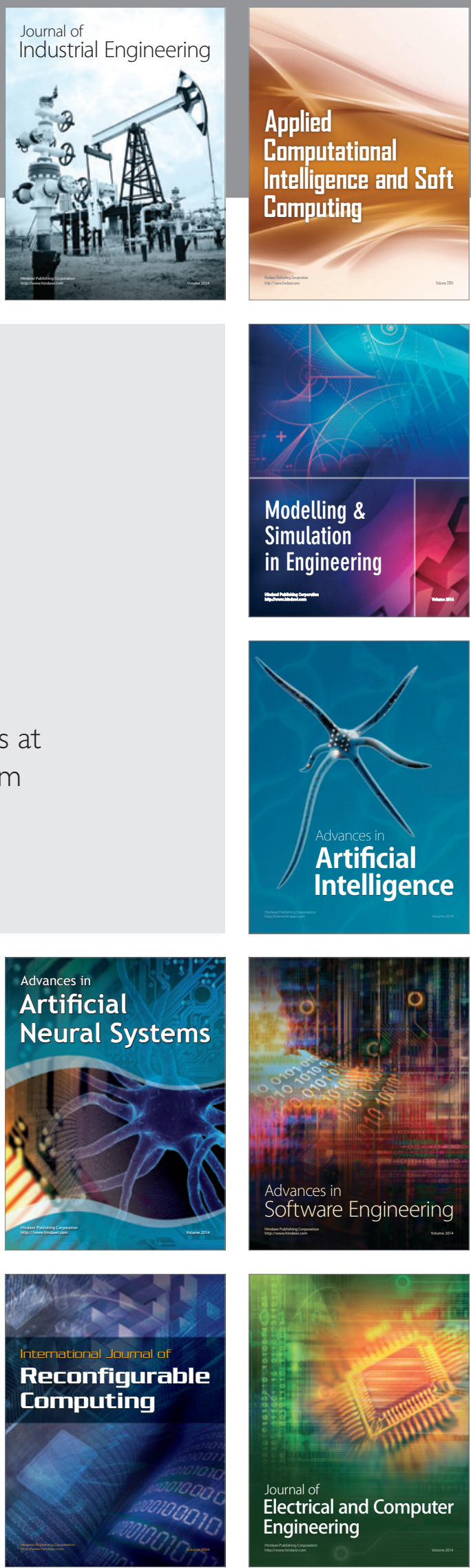